\title{
Treatment of Recurrent Hemarthrosis after Total Knee Arthroplasty
}

\author{
Ju-Hyung Yoo, MD, Hyun-Cheol Oh, MD, Sang-Hoon Park, MD, Sanghyeon Lee, MD, Yunjae Lee, MD, \\ and Seong-Hun Kim, MD \\ Department of Orthopedic Surgery, National Health Insurance Service Ilsan Hospital, Goyang, Korea
}

\begin{abstract}
Purpose: The purpose of this study is to evaluate the incidence and treatment of recurrent hemarthrosis after total knee replacement (TKR).
Materials and Methods: Among a total of 5,510 patients who underwent TKR from March 2000 to October 2016, patients who had two or more bleeding 2 weeks after surgery were studied. Conservative treatments were performed for all cases with symptoms. In patients who did not respond to conservative treatment several times, embolization was performed. We retrospectively evaluated the postoperative bleeding time, bleeding frequency, treatment method, and outcome.

Results: Seventeen $(0.3 \%)$ of the 5,510 patients developed recurrent hemarthrosis. Bleeding occurred at an average of 2 years 3 months after the operation. Joint aspiration was performed 3.5 times (range, 2 to 10 times) on average, and 14 cases (82.3\%) were treated with conservative treatment. In 3 patients with severe bleeding and hemorrhage, embolization was performed.

Conclusions: Recurrent hemarthrosis after TKR is a rare disease with a low incidence of $0.3 \%$ and usually could be treated by conservative treatment. If recurrences occur repeatedly, embolization through angiography or surgical treatment may be considered, but the results are not satisfactory and careful selection of treatment modalities is warranted.
\end{abstract}

Keywords: Knee, Arthroplasty, Hemarthrosis, Embolization

\section{Introduction}

The prevalence of total knee replacement (TKR) has been increasing in the aging society since 1980s, and development of advanced surgical techniques has contributed to improvement in satisfaction after surgical treatment; however, it is of note that the incidence of complications is also growing as the frequency of TKR increases ${ }^{1,2}$. Recurrent hemarthrosis is a rare complication of TKR with a prevalence of $0.3 \%-0.65 \%^{3-6)}$. The reported mean interval from TKR to the onset of hemarthrosis is 2 years, which

Received July 29, 2017; Revised (1st) October 12, 2017;

(2nd) November 3, 2017; Accepted December 2, 2017

Correspondence to: Seong-Hun Kim, MD

Department of Orthopedic Surgery, National Health Insurance Service Ilsan Hospital, 100 Ilsan-ro, Ilsandong-gu, Goyang 10444, Korea

Tel: +82-31-900-0436, Fax: +82-31-900-0343

E-mail: esmeron@naver.com

This is an Open Access article distributed under the terms of the Creative Commons Attribution Non-Commercial License (http://creativecommons.org/licenses/by-nc/4.0/) which permits unrestricted non-commercial use, distribution, and reproduction in any medium, provided the original work is properly cited. ranges from 2 weeks to 18 years ${ }^{3,4,7,8)}$

Recurrent bleeding in the joint can result in joint stiffness, poor postoperative function, and, albeit rare, deep joint sepsis ${ }^{9,10)}$. Thus, proper treatment is imperative. The etiology of recurrent hemarthrosis documented in the literature includes repetitive trauma to the hypervascular hypertrophied synovium ${ }^{6}$, impingement of the hypertrophied synovium between the femoral and tibial components ${ }^{3,4,7)}$, pigmented villonodular synovitis ${ }^{11,12)}$, anticoagulant therapy ${ }^{7)}$, intra- or extra-articular tumor growth, pseudoaneurysm, arteriovenous fistulae ${ }^{10,13-16)}$, a femoral component eroding through a hypertrophied atherosclerotic artery ${ }^{17}$, bleeding from the arterial branches ${ }^{6,18)}$, bleeding disorders such as hemophilia ${ }^{19)}$, implant malalignment or instability, and polyethylene wear, ${ }^{6,17)}$. However, some cases have obscure causes that disrupt effective treatment.

Recurrent hemarthrosis can be treated conservatively (joint aspiration, compression, ice packs, and rest) or surgically (interventional angiographic embolization, radiosynovectomy ${ }^{20)}$, and arthroscopic/open synovectomy). However, a standard treatment method has not yet been established. In this study, we set out to 
investigate the incidence of recurrent hemarthrosis after TKR and appropriate treatment methods.

\section{Materials and Methods}

This study was approved by the Institutional Review Board of the Ethics Committee of the National Health Insurance Service Ilsan Hospital. From March 2000 to October 2016, 5,510 TKRs were performed at our institution by the first author (JHY) of this study. Of those, cases with two or more bleeding episodes from 2 weeks after surgery were included in this study. The surgery was performed using LCS system (DePuy, Warsaw, IN, USA) in 181 shoulders, Maxim complex knee system (Biomet, Warsaw, IN, USA) in 76 shoulders, and Nexgen Legacy Posterior Stabilized Flex Fixed Bearing (LPS Flex Fixed; Zimmer, Warsaw, IN, USA) in 5,253 shoulders. Until July 2004, a conventional approach was used in the surgery in 326 shoulders; thereafter, minimally invasive surgery (MIS) using MIS Quad-Sparing Instrumentation (Zimmer) was performed in 5,184 shoulders.

For symptomatic patients, conservative treatment was the first treatment option, which involved joint aspiration, compression, rest, ice pack application, and splinting, and antithrombotic therapy was discontinued. For recurrent hemarthrosis in spite of three or more joint aspiration sessions, interventional embolization was performed upon confirmation of bleeding with interventional angiography. For bleeding after embolization, arthrotomy was carried out to identify the bleeding source. The interval between TKR and the onset of bleeding, frequency of bleeding, and treatment method and outcome were retrospectively evaluated.

Table 1. Data of Patients

\begin{tabular}{|c|c|c|c|c|c|c|c|c|c|c|}
\hline No. & Sex & $\begin{array}{l}\text { Age } \\
(\mathrm{yr})\end{array}$ & Site & $\begin{array}{c}\text { BMI } \\
\left(\mathrm{kg} / \mathrm{m}^{2}\right)\end{array}$ & Anticoagulant & $\begin{array}{l}\text { Interval between TKA } \\
\text { and hemarthrosis (wk) }\end{array}$ & Aspiration & $\begin{array}{c}\text { Angio \& } \\
\text { embolization }\end{array}$ & $\begin{array}{c}\text { Surgical } \\
\text { exploration }\end{array}$ & Results \\
\hline 1 & $\mathrm{~F}$ & 65 & $\mathrm{~L}$ & 26.3 & - & 70 & 7 & Yes & Yes & Improved \\
\hline 2 & $\mathrm{~F}$ & 68 & $\mathrm{R}$ & 25.4 & - & 238 & 4 & No & No & Improved \\
\hline 3 & $\mathrm{~F}$ & 70 & $\mathrm{R}$ & 26.2 & Aspirin & 310 & 3 & No & No & Improved \\
\hline 4 & $\mathrm{~F}$ & 71 & $\mathrm{~L}$ & 27.5 & $\begin{array}{l}\text { Arixtra (fondaparinux } \\
\text { sodium) }\end{array}$ & 54 & 2 & No & No & Improved \\
\hline 5 & $\mathrm{~F}$ & 70 & $\mathrm{~L}$ & 24.5 & - & 67 & 4 & No & No & Improved \\
\hline 6 & $\mathrm{~F}$ & 66 & $\mathrm{R}$ & 36.7 & Aspirin & 158 & 4 & No & No & Improved \\
\hline 7 & $\mathrm{~F}$ & 71 & $\mathrm{R}$ & 29.9 & Aspirin & 404 & 3 & No & No & Improved \\
\hline 8 & $\mathrm{~F}$ & 67 & $\mathrm{R}$ & 25.4 & $\begin{array}{l}\text { Arixtra (fondaparinux } \\
\text { sodium) \& Ginkgo } \\
\text { biloba extract }\end{array}$ & 98 & 5 & Yes & No & Improved \\
\hline 9 & M & 76 & $\mathrm{R}$ & 22.8 & Wafarin & 2 & 2 & No & No & Improved \\
\hline 10 & $\mathrm{~F}$ & 61 & $\mathrm{R}$ & 23.8 & $\begin{array}{l}\text { Arixtra (fondaparinux } \\
\text { sodium) }\end{array}$ & 25 & 2 & No & No & Improved \\
\hline 11 & $\mathrm{~F}$ & 75 & $\mathrm{~L}$ & 27.2 & Arixtra (rivaroxaban) & 166 & 2 & No & No & Improved \\
\hline 12 & $\mathrm{~F}$ & 63 & $\mathrm{R}$ & 25.4 & Rivaroxaban & 139 & 2 & No & No & Improved \\
\hline 13 & M & 65 & $\mathrm{~L}$ & 28.0 & Aspirin & 2 & 10 & Yes & Yes & $\begin{array}{l}\text { Improved } \\
\text { after another } \\
\text { embolization }\end{array}$ \\
\hline 14 & F & 70 & $\mathrm{~L}$ & 22.1 & - & 2 & 2 & No & No & Improved \\
\hline 15 & M & 76 & $\mathrm{R}$ & 21.2 & - & 8 & 4 & No & No & Improved \\
\hline 16 & $\mathrm{~F}$ & 76 & $\mathrm{~L}$ & 22.0 & $\begin{array}{l}\text { Arixtra (fondaparinux } \\
\text { sodium) }\end{array}$ & 65 & 2 & No & No & Improved \\
\hline 17 & $\mathrm{~F}$ & 65 & $\mathrm{~L}$ & 32 & $\begin{array}{l}\text { Arixtra (fondaparinux } \\
\text { sodium) \& aspirin }\end{array}$ & 79 & 2 & No & No & Improved \\
\hline
\end{tabular}

BMI: body mass index, TKA: total knee arthroplasty, L: left, R: right. 


\section{Results}

The incidence of recurrent hemarthrosis was 17 of 5,510 shoulders $(0.3 \%)$ (Table 1$)$. The mean age of the patients with hemarthrosis was 69.1 years (range, 61 to 76 years). There were 3 males and 14 females. The affected shoulder was the right side in 9 cases and left side in 8 cases. At the time of bleeding, 12 patients

Table 2. Demographics of Patients of Recurrent Hemarthrosis

\begin{tabular}{lc}
\hline \multicolumn{1}{c}{ Recurrent hemarthrosis } & Value \\
\hline No. of cases (\%) & $17(0.3)$ \\
Gender & 3 \\
Male & 14 \\
Female & $69.1(61-76)$ \\
Age (yr), mean (range) & $26.3 \pm 3.9$ \\
BMI (kg/m ${ }^{2}$ ), mean \pm SD & \\
Preoperative diagnosis & 17 \\
Osteoarthritis & $12(70.5)$ \\
Anticoagulant medication (\%) & 4 \\
Aspirin & 2 \\
Antiplatelet agent & 5 \\
Aspirin+antiplatelet agent & 1 \\
Warfarin & \\
Implant & 17 \\
Nexgen (case) & \\
Surgical approach & 17 \\
MIS technique (case) &
\end{tabular}

BMI: body mass index, SD: standard deviation, MIS: minimally invasive surgery. were on antithrombotic therapy using aspirin in 4, antiplatelets in 2 , aspirin+antiplatelets in 5 , and warfarin in 1 . The preoperative diagnosis was osteoarthritis in all 17 cases. All knees received Nexgen prosthesis in TKR performed via a minimally invasive approach (Table 2). No patient had a history of trauma at the time of bleeding. The femoral and tibial component alignment was normal on radiographs. Component loosening or migration, osteolysis, or joint instability was not observed (Table 3 ).

The interval between bleeding and TKR was an average of 2 years and 3 months (116 weeks; range, 2 weeks to 7 years and 9 months). Joint aspiration was performed 3.5 times on average (range, 2 to 10 times). Conservative treatment was effective for symptomatic improvement in 14 cases (82.3\%). Three cases refractory to conservative treatment and 3 sessions of joint aspiration underwent interventional angiography and embolization. In the first case, angiography revealed bleeding from the superior lateral geniculate artery. In spite of embolization of the artery,

Table 3. Radiological Data

\begin{tabular}{lc}
\hline \multicolumn{1}{c}{ Characteristic } & Value \\
\hline Tibial component alignment angle $\left(^{\circ}\right)$ & $0.8 \pm 1.3$ varus \\
Tibial component posterior inclination $\left(^{\circ}\right)$ & $2.9 \pm 1.9$ \\
Femorotibial angle $\left(^{\circ}\right)$ & $5.8 \pm 2.0$ valgus \\
Tibial component alignment angle in $0^{\circ} \pm 3^{\circ}(\%)$ & 94 \\
Femorotibial angle in $6^{\circ} \pm 3^{\circ}(\%)$ & 94 \\
Loosening (case) & 0 \\
Osteolysis (case) & 0 \\
Migration (case) & 0 \\
\hline
\end{tabular}
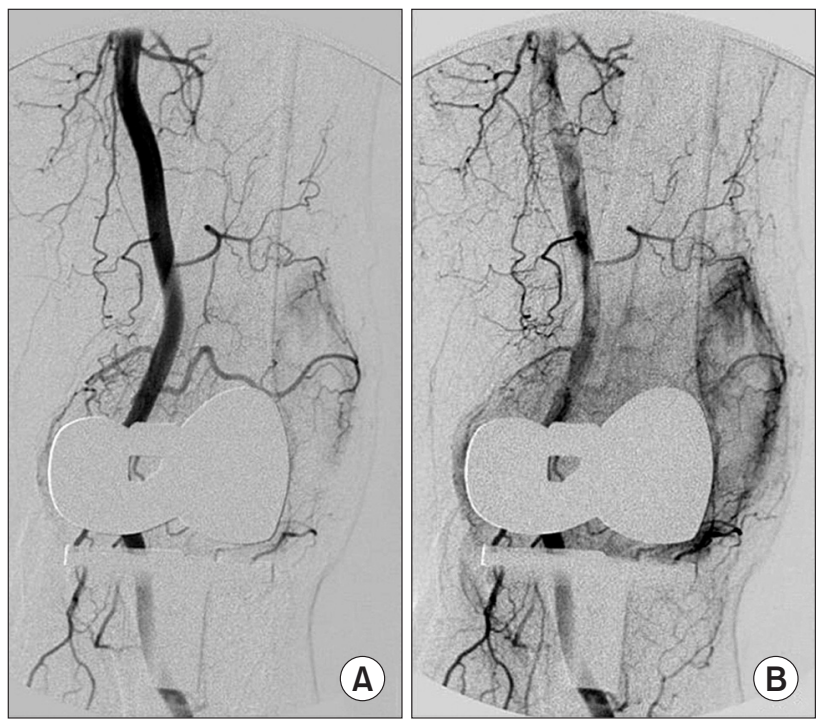

Fig. 1. (A) Interventional angiography was performed in the left lower limb in a 67-year-old woman with recurrent hemarthrosis. (B) In angiography, a vascular blush was observed in the left superior lateral geniculate artery. (C) Successful embolization was done using 1:4 mixture of glue and lipiodol for the left superior lateral geniculate artery (arrow). However, bleeding from the inferior lateral geniculate artery (arrow head) was neglected. 

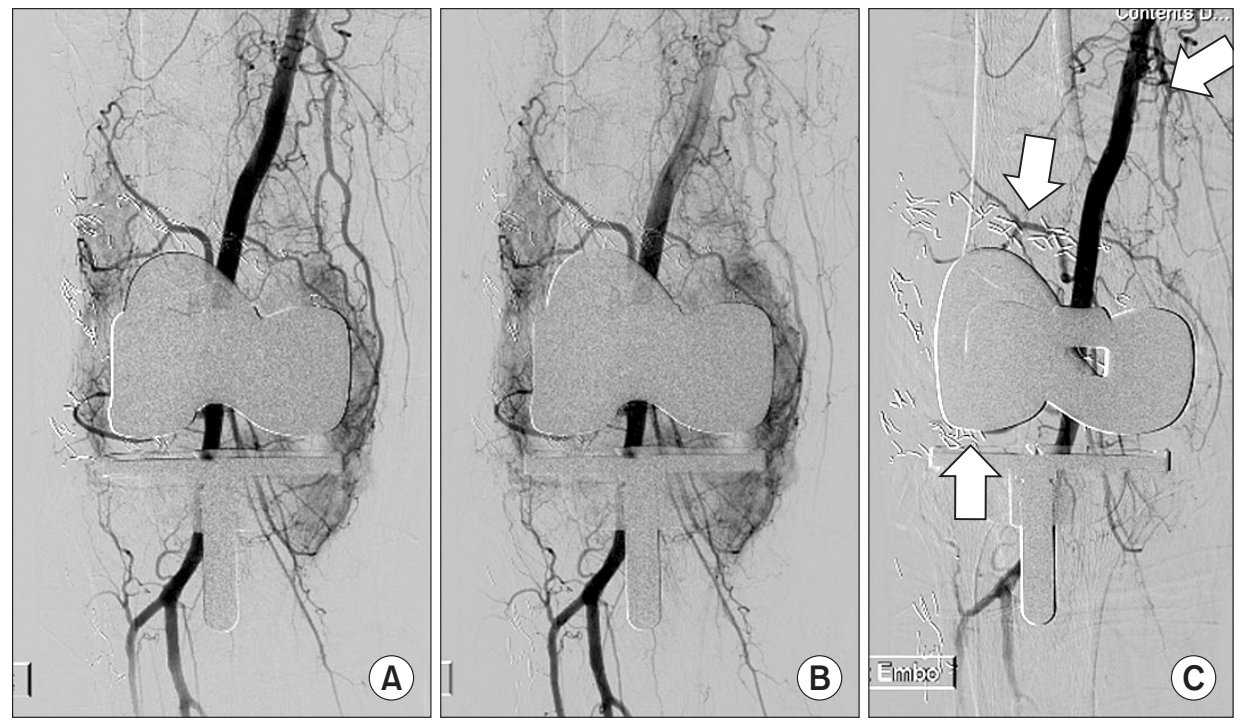

Fig. 2. (A) Interventional angiography was performed in the right lower limb in a 69-year-old woman with recurrent hemarthrosis. (B) In angiography, a vascular blush was observed in the right superior and inferior lateral geniculate arteries and descending geniculate artery. (C) Gelfoam embolization was performed for the right superior and inferior lateral geniculate arteries and descending geniculate artery (arrows). bleeding recurred. Through arthrotomy, the source of bleeding was found in the inferior lateral geniculate artery and controlled by hemostasis (Fig. 1). In the second case, recurrence was reported once 2 years after embolization of the superior and inferior lateral geniculate arteries and descending geniculate artery, but it did not lead to another episode of bleeding (Fig. 2). In the third case, bleeding recurred after embolization of the superior and inferior lateral geniculate arteries. During subsequently performed arthrotomy, the source of bleeding was not identified. After discharge, the patient was not available for follow-up at our institution. At another hospital, the patient underwent 3 sessions of joint aspiration due to recurrence. After embolization performed at the hospital, bleeding has not recurred for three years.

\section{Discussion}

Recurrent hemarthrosis following TKR is a rare complication; it was observed in only $17(0.3 \%)$ of the total 5,510 TKRs in this study. Thus, it is challenging to determine the cause and prescribe proper treatment. Most hemarthrosis patients complain of acute pain and swelling of the knee with some loss of function without any trauma ${ }^{7,11,21)}$. Due to the difficulty of identifying the cause of such symptoms, conservative treatment is initially employed to halt further hemorrhage, which involves joint aspiration, rest, ice pack application, and splinting. It is also advised to discontinue the use of anticoagulants, unless required for the patient's medical condition. Further, it is necessary to investigate the presence of coagulation disorder by assessing the prothrombin time, activated thromboplastin time, and bleeding time and to evaluate the platelet function based on the thrombin consumption index, Von
Wellebrand factor level, platelet factor 3 availability, and platelet aggregation study ${ }^{9}$. Conservative treatment resulted in improvement in 9 of 30 patients in a study by Kindsfater and Scott ${ }^{7}$ and in 3 of 10 patients in a study by Ohdera et al. ${ }^{4}$. In the current study, 14 (82.3\%) of 17 patients obtained improvement with conservative treatment alone. For recurrent hemarthrosis following failing conservative therapy, magnetic resonance angiography ${ }^{22}$, interventional angiography \& embolization, radiosynovectomy (synoviorthesis) $^{20)}$, and arthroscopic/open arthrotomy can be considered. Magnetic resonance angiography ${ }^{22}$ is a non-invasive modality for the identification of bleeding source and interventional treatment.

Recurrent hemarthrosis after TKR can be characterized as intermittent and acute bleeds in most cases. The most probable cause is bleeding from the peripheral branches of the artery. Interventional angiography \& embolization can be the ultimate solution for this, which will produce desirable effects when performed as soon as possible. Angiography allows for identification of "blush-type" hypervascular synovium, vascular abnormality, and vascular damage as well as embolization for treatment. Stent implantation can also be performed for pseudoaneurysm or arteriovenous fistulae. Although it carries the risk of radiation exposure, iatrogenic vascular injury, and contrast-induced nephropathy, it can be performed under local anesthesia, lowers the risk of infection, and expedites postoperative recovery compared to open surgery ${ }^{23)}$. The most common source of bleeding confirmed with angiography is the superior lateral geniculate artery ${ }^{14,15,17,23,24)}$, followed by the superior medial geniculate artery ${ }^{23)}$ and the inferior lateral geniculate artery ${ }^{21,23)}$, and the characteristic feature is "blush-type" synovial hypervascularity. On the 
treatment outcomes of embolization for hemarthrosis after TKR, Weinder et al. ${ }^{24)}$ reported that $12(92.3 \%)$ of 13 patients obtained resolution of hemarthrosis after one session of embolization, and another session of embolization resulted in resolution in the remaining 1 case. Tat-Sing Law and $\mathrm{McClure}^{25)}$ reported improvement in all three cases treated by angiography and embolization.

In our study, angiography performed in the three cases revealed "blush-type" synovial hypervascularity, but vascular malformation or pseudoaneurysm was not observed. In the first case, we initially performed embolization of the superior lateral geniculate artery in angiography, which led to recurrence. With arthrotomy, bleeding from a branch of the inferior lateral geniculate artery was confirmed. Based on a review of the record of previous angiography, the recurrence was attributed to failure to detect the bleeding source and embolize the inferior lateral geniculate artery. In the second case, we performed embolization of the inferior/superior lateral geniculate artery branches. In spite of one episode of bleeding 2 years after surgery, no recurrence has been reported afterwards. In the third case, we performed angiography and embolization of the superior/inferior lateral geniculate arteries after 10 episodes of bleeding, which failed to resolve bleeding. Subsequently, arthrotomy was carried out, but the source of bleeding was not identified during the procedure. Only after angiography and embolization performed at another hospital, the patient could obtain improvement.

Considering that hemorrhage occurs intermittently, rather than continuously, appropriate timing for angiographic embolization is crucial for treatment success of hemarthrosis. In addition, the various possible sources of bleeding including the inferior/superior lateral geniculate artery branches, inferior/superior medial geniculate artery branches, and other areas should be thoroughly examined. For a highly suspected source of bleeding, thorough embolization is advised. One of the common non-surgical treatment options is intra-articular injection of Yttrium 90-citrate to reduce the hypertrophied synovium and control bleeding since hypertrophic synovium is responsible for hemarthrosis in most cases ${ }^{20)}$.

Surgical treatment can be performed arthroscopically, which is advantageous for less soft tissue damage and earlier rehabilitation than open surgery; however, the limited surgical field of view may restrict identification of the source of bleeding and proper treatment, increasing the risk of recurrence ${ }^{7)}$. Arthroscopic arthrotomy can be considered for recurrent hemarthrosis refractory to conservative treatment or angiographic embolization. Common intraoperative findings include hypervascular, proliferative synovium and impingement of synovial tissues between femoral and tibial components. However, it is difficult to locate the exact source of bleed- ing. Also, the etiology of hypervascular, proliferative synovium has not be clearly elucidated. It has been postulated that intraarticular bleeding stimulates production and activation of inflammatory mediators in the joint, which results in the occurrence of synovitis and proliferation of blood vessels in the synovium, thus rendering it susceptible to trauma and hemorrhage ${ }^{6,26)}$. Symptomatic improvement can be expected with synovectomy alone in many cases even without identification of the source of bleeding in arthrotomy ${ }^{3-5)}$. Worland and Jessup ${ }^{3)}$ reported complete healing in 7 cases after arthrotomy. In the current study, bleeding recurred after angiography in the first case. In the following arthrotomy, the source of bleeding was identified almost at the end of the procedure during probe manipulation around the inferior lateral geniculate artery. Therefore, considering that temporary contraction of blood vessels during arthrotomy may disrupt early detection of the source of bleeding, it is advised to thoroughly examine the suspected region with a probe.

One of the limitations of this study is the lack of comparison of different treatment methods because treatment was carried out without a protocol clearly established. In addition, in spite of the large enrollment, statistical significance was difficult to determine due to the low incidence of recurrent hemarthrosis in the enrolled patients. In an effort to overcome this limitation, we assessed the incidence among a large consecutive number of cases $(5,510)$ performed by a single surgeon over a period of 16 years. Based on our findings, we believe that conservative treatment, interventional angiographic embolization, and surgical treatment can all be effective for the treatment of recurrent hemarthrosis.

\section{Conclusions}

Recurrent hemarthrosis following TKR is rare disease with a low incidence of $0.3 \%$. It can be treated conservatively in most cases, for which appropriate pressure application, rest, and sufficient period of immobilization after joint aspiration are essential. Angiography can be considered for recurrent bleeding refractory to conservative treatment for thorough identification of the source of bleeding and embolization. If hemarthrosis recurs even after embolization, arthrotomy may be necessary. However, a cautious approach should be taken with regard to selection of treatment modalities due the difficulty of locating the source of bleeding.

\section{Conflict of Interest}

No potential conflict of interest relevant to this article was re- 
ported.

\section{References}

1. Dixon T, Shaw M, Ebrahim S, Dieppe P. Trends in hip and knee joint replacement: socioeconomic inequalities and projections of need. Ann Rheum Dis. 2004;63:825-30.

2. Healy WL, Della Valle CJ, Iorio R, Berend KR, Cushner FD, Dalury DF, Lonner JH. Complications of total knee arthroplasty: standardized list and definitions of the Knee Society. Clin Orthop Relat Res. 2013;471:215-20.

3. Worland RL, Jessup DE. Recurrent hemarthrosis after total knee arthroplasty. J Arthroplasty. 1996;11:977-8.

4. Ohdera T, Tokunaga M, Hiroshima S, Yoshimoto E, Matsuda S. Recurrent hemarthrosis after knee joint arthroplasty: etiology and treatment. J Arthroplasty. 2004;19:157-61.

5. Oishi CS, Elliott ML, Colwell CW Jr. Recurrent hemarthrosis following a total knee arthroplasty. J Arthroplasty. 1995;10 Suppl:S56-8.

6. Saksena J, Platts AD, Dowd GS. Recurrent haemarthrosis following total knee replacement. Knee. 2010;17:7-14.

7. Kindsfater K, Scott R. Recurrent hemarthrosis after total knee arthroplasty. J Arthroplasty. 1995;10 Suppl:S52-5.

8. Bagla S, Rholl KS, van Breda A, Sterling KM, van Breda A. Geniculate artery embolization in the management of spontaneous recurrent hemarthrosis of the knee: case series. J Vasc Interv Radiol. 2013;24:439-42.

9. Malhotra R, Bhan S, Kiran EK. Haemarthroses after total knee arthroplasty caused by an isolated platelet factor 3 availability defect. J Bone Joint Surg Br. 2005;87:1549-52.

10. Sharma H, Singh GK, Cavanagh SP, Kay D. Pseudoaneurysm of the inferior medial geniculate artery following primary total knee arthroplasty: delayed presentation with recurrent haemorrhagic episodes. Knee Surg Sports Traumatol Arthrosc. 2006;14:153-5.

11. Ballard WT, Clark CR, Callaghan JJ. Recurrent spontaneous hemarthrosis nine years after a total knee arthroplasty: a presentation with pigmented villonodular synovitis. J Bone Joint Surg Am. 1993;75:764-7.

12. Hamlin BR, Duffy GP, Trousdale RT, Morrey BF. Total knee arthroplasty in patients who have pigmented villonodular synovitis. J Bone Joint Surg Am. 1998;80:76-82.

13. Haddad FS, Prendergast CM, Dorrell JH, Platts AD. Arteriovenous fistula after fibular osteotomy leading to recurrent haemarthroses in a total knee replacement. J Bone Joint Surg Br. 1996;78:458-60.
14. Ibrahim M, Booth RE Jr, Clark TW. Embolization of traumatic pseudoaneurysms after total knee arthroplasty. J Arthroplasty. 2004;19:123-8.

15. Katsimihas M, Robinson D, Thornton M, Langkamer VG. Therapeutic embolization of the genicular arteries for recurrent hemarthrosis after total knee arthroplasty. J Arthroplasty. 2001;16:935-7.

16. Kalsi PS, Carrington RJ, Skinner JS. Therapeutic embolization for the treatment of recurrent hemarthrosis after total knee arthroplasty due to an arteriovenous fistula. J Arthroplasty. 2007;22:1223-5.

17. Cunningham RB, Mariani EM. Spontaneous hemarthrosis 6 years after total knee arthroplasty. J Arthroplasty. 2001;16: 133-5.

18. Dhondt E, Vanhoenacker FM, D' Archambeau O, Snoeckx A, Defreyne L. Angiographic findings and therapeutic embolization of late hemarthrosis after total joint arthroplasty. Skeletal Radiol. 2009;38:31-6.

19. Silva M, Luck JV Jr. Long-term results of primary total knee replacement in patients with hemophilia. J Bone Joint Surg Am. 2005;87:85-91.

20. Kapetanos GA, Papavasiliou KA, Makris V, Nikolaides AP, Kirkos JM, Symeonides PP. Recurrent spontaneous hemarthrosis after total knee arthroplasty successfully treated with synoviorthesis. J Arthroplasty. 2008;23:931-3.

21. Rukavina A, Kerkhoffs GM, Schneider P, Kuster MS. Recurrent hemarthrosis after total knee arthroplasty. Knee Surg Sports Traumatol Arthrosc. 2010;18:898-900.

22. Hash TW 2nd, Maderazo AB, Haas SB, Saboeiro GR, Trost DW, Potter HG. Magnetic resonance angiography in the management of recurrent hemarthrosis after total knee arthroplasty. J Arthroplasty. 2011;26:1357-61.

23. Maheshwari R, Kelley SP, Langkamer VG, Loveday E. Spontaneous recurrent haemarthrosis following unicompartmental knee arthroplasty and its successful treatment by coil embolisation. Knee. 2004;11:413-5.

24. Weidner ZD, Hamilton WG, Smirniotopoulos J, Bagla S. Recurrent hemarthrosis following knee arthroplasty treated with arterial embolization. J Arthroplasty. 2015;30:2004-7.

25. Tat-Sing Law M, McClure DN. Therapeutic embolization in the treatment of recurrent haemarthrosis following knee arthroplasty. ANZ J Surg. 2010;80:247-9.

26. Pritsch T, Pritsch M, Halperin N. Therapeutic embolization for late hemarthrosis after total knee arthroplasty: a case report. J Bone Joint Surg Am. 2003;85:1802-4. 